\title{
Risk of Dementia in Long-Term Benzodiazepine Users: Evidence from a Meta-Analysis of Observational Studies
}

\author{
Qian $\mathrm{He}^{\mathrm{a}}$ \\ Xiaohua Chen ${ }^{\mathrm{b}}$ \\ Tang $\mathrm{Wu}^{\mathrm{a}}$ \\ Liyuan $\mathrm{Li}^{\mathrm{a}}$ \\ Xiaofan $\mathrm{Fei}^{\mathrm{c}}$ \\ ${ }^{a}$ Departments of Out-Patient, \\ ${ }^{b}$ Nursing, and ${ }^{\mathrm{C}}$ Pharmacy, \\ West China Hospital, Sichuan Uniwersity, \\ Chengdu, China
}

\begin{abstract}
Background and Purpose There is conflicting evidence in the literature on the association between benzodiazepines (BDZs) and the risk of dementia. This meta-analysis aimed to determine the relationship between the long-term usage of BDZs and the risk of dementia.

Methods The PubMed and Embase databases were systematically searched for relevant publications up to September 2017. The literature search focused on observational studies that analyzed the relationship between the long-term use of BDZs and the risk of dementia. Pooled rate ratios (RRs) with 95\% confidence interval (CI) were assessed using a random-effects model. The robustness of the results was checked by performing subgroup and sensitivity analyses.

Results Ten studies were included: six case-control and four cohort studies. The pooled RR for developing dementia was 1.51 (95\% $\mathrm{CI}=1.17-1.95, p=0.002)$ in patients taking BDZ. The risk of dementia was higher in patients taking $\mathrm{BDZ}$ s with a longer half-life $(\mathrm{RR}=1.16,95 \% \mathrm{CI}=$ $0.95-1.41, p=0.150)$ and for a longer time $(\mathrm{RR}=1.21,95 \% \mathrm{CI}=1.04-1.40, p=0.016)$.

Conclusions This meta-analysis that pooled ten studies has shown that BDZ significantly increases the risk of dementia in the elderly population. The risk is higher in patients taking BDZ with a longer half-life ( $>20$ hours) and for a longer duration ( $>3$ years).

Key Words dementia, benzodiazepines, meta-analysis.
\end{abstract}

\section{INTRODUCTION}

Benzodiazepines (BDZs) enhance the efficacy of the neurotransmitter gamma-aminobutyric acid (GABA) at the GABA-A receptor so as to induce sedative, hypnotic, anxiolytic, anticonvulsant, and muscle relaxant properties. ${ }^{1}$ Various studies have been conducted to understand the association between $\mathrm{BDZ}$ and dementia. ${ }^{2-11} \mathrm{~A}$ few well-conducted prospective cohort studies have found an increased risk of dementia in users of long-acting BDZ, whereas subsequent studies found no such association.

The type of BDZ activity (long acting or short acting) and the time period of taking the drug (long term or short term) were also considered in previous analyses. A few studies have inferred that the long-term use of $\mathrm{BDZ}$ could increase the risk of dementia, whereas its shortterm use might not. ${ }^{2,8,10}$ Moreover, long-acting BDZ but not short-acting BDZ might be related to an increased risk of dementia. ${ }^{7,9,11}$

To resolve these discrepancies, the current pooled meta-analysis was designed to establish the association between the long-term use of $\mathrm{BDZ}$ and the risk of dementia, in terms of both the duration of action and type of BDZ taken.

$(\circledast)$ This is an Open Access article distributed under the terms of the Creative Commons Attribution Non-Commercial License (https://creativecommons.org/licenses/by-nc/4.0) which permits unrestricted non-commercial use, distribution, and reproduction in any medium, provided the original work is properly cited. 


\section{METHODS}

\section{Search strategy}

A systematic search strategy of the following electronic databases was used to identify all published studies on the association between $\mathrm{BDZ}$ use and the risk of dementia: Medline (via Ovid), Embase, and the Cochrane Library. The search terms and keywords were altered in accordance with the specifications of the individual databases. The search strategies are given in the Supplementary Material 1 (in the online-only Data Supplement). No restrictions were placed on publication language. The database search was performed on January 22, 2018. The reference lists of identified articles were checked for additional publications, while the corresponding authors were contacted to obtain additional information about both published and unpublished studies. The present study was reported according to PRISMA guidelines for reporting metaanalyses.

\section{Study selection}

The results obtained from searching the three databases were exported into EndNote X8.0.1 software to identify and remove potential duplicate studies that appeared in more than one database. All of the resulting unique studies were exported into an Excel spreadsheet for initial screening, which involved the titles and/or Abstracts of the unique studies in Excel being checked by two authors (T.W. and L.L.) to exclude any clearly irrelevant studies. For secondary screening, the full text was read to decide on inclusion or exclusion by two authors (T.W. and X.C.) independently based on the selection criteria of the meta-analysis. Disagreement was resolved by a third author (Q.H.) who examined the studies independently.

\section{Inclusion and exclusion criteria}

Studies were eligible for inclusion if they met the following inclusion criteria: 1) a case-control or cohort design, 2) exposure of interest was $\mathrm{BDZ}$ intake, 3) outcome was incidence of dementia, and 4) relative risks or odds ratios (ORs) with corresponding 95\% confidence interval (CI) reported or could be estimated (from the raw data in the published article).

\section{Data extraction}

The primary studies were reviewed by two authors independently to evaluate their relevance for inclusion in the current pooled analysis. The following data points were then extracted for each study: 1) author name, publication year, and study site, 2) study design, 3) number of study subjects, and numbers of study subjects on $\mathrm{BDZ}$ and having dementia, 4) effect estimates with CIs, 5) assessment of exposure (BDZ us- age) and outcome (dementia), and 6) confounding factors adjusted, if applicable.

\section{Quality assessment}

Any inconsistencies in opinion between the two independent authors were resolved by an analysis of the published article by a third author. The Newcastle-Ottawa Scale (NOS) was used to measure the quality of each study. This scale considers the three factors of selection (4 points), comparability (2 points), and outcome/exposure (3 points), with the study quality being highest for the maximum score of 9 points, moderate for a score of 7 or 8 points, and low for a score $\leq 6$ points.

\section{Data synthesis and analysis}

The rate ratio (RR) was used as the effect estimate in pooling. Both cohort and case-control studies were allowed since the risk of dementia is low and the $\mathrm{RR}$ in prospective cohort studies will mathematically approximate the OR. A random-effects model (the DerSimonian-Laird method) was chosen for pooling the effect estimates of individual studies, since it was assumed that the effect sizes underlying different studies are drawn from a distribution rather than representing a common effect size shared by all studies. The overall pooled $\mathrm{RR}$ estimate was calculated by comparing ever BDZ users with never $\mathrm{BDZ}$ users. Heterogeneity was assessed in various ways, including visual examination of a forest plot, the Cochrane $Q$ test, and the $I^{2}$ statistic. A Q statistic with a $p$ value of $<0.01$ and $\mathrm{I}^{2}$ value $>50 \%$ was considered to indicate heterogeneity. Subgroup analyses were performed to assess the sources of heterogeneity according to study design (cohort vs. casecontrol studies) and study quality.

A secondary analysis was performed to determine the effects on dementia of the duration of BDZ usage (effect estimate for long-term usage was obtained by comparing longterm $\mathrm{BDZ}$ usage with short-term $\mathrm{BDZ}$ usage) and the half-life of $\mathrm{BDZ}$ (effect estimate for the use of long-acting BDZ was obtained by comparing the use of long-acting $\mathrm{BDZ}$ with the use of other types of BDZ: ultra-short-, short-, and mediumterm-acting BDZ). A subgroup analysis could not be conducted based on the duration of $\mathrm{BDZ}$ use and BDZ pharmacokinetics since these are not primary estimates and these effect estimates are not reported for all studies. Therefore, a secondary pooled analysis was performed to draw further inferences on the use of BDZ.

The test for interaction developed by Altman and Bland ${ }^{12}$ is useful for assessing if there is a significant difference between two effect estimates (E1 and E2) obtained from a subgroup analysis. The result of the test for interaction is interpreted based on the $p_{\text {interaction }}$ value, and the $\mathrm{z}$ value is calculated as: 
erence lists of the included articles (Fig. 1). We excluded 358 duplicates using EndNote reference manager and 2,623 studies found to be ineligible after reading titles and Abstracts. Accordingly, we retrieved 49 references for further assessment. We excluded 39 references since 20 did not report separate data on BDZs, 16 studies did not assess the casual relationship between BDZ use and dementia, and 3 did not report original data. The ten studies remaining for inclusion in the meta-analysis comprised four cohort and six case-control studies.

\section{Study characteristics}

The 4 cohort studies (Table 1 ) and 6 case-control studies (Table 2) involved 171,939 subjects and 42,025 dementia cases. The follow-up period was 4-22 years in these studies, and the publication period was 2002-2017. The mean age of patients in all of the included studies was older than 70 years, with the exception of Gallacher et al. ${ }^{2}$ reporting a mean age of 61 years. This meant that all of the included results were for elderly patients; the mean ages in the individual studies are presented in Table 1 and 2. The studies included more females than males, with the exception of Gallacher et al. ${ }^{2}$ studying male patients only.

The 6 case-control studies involved 159,124 participants

\section{Search results}

The 3,030 references identified included 3,026 articles (258 from Medline, 2,551 from Embase, and 217 from the Cochrane Library) found by searching the electronic databases up to January 2018, plus 4 articles found by screening the ref-

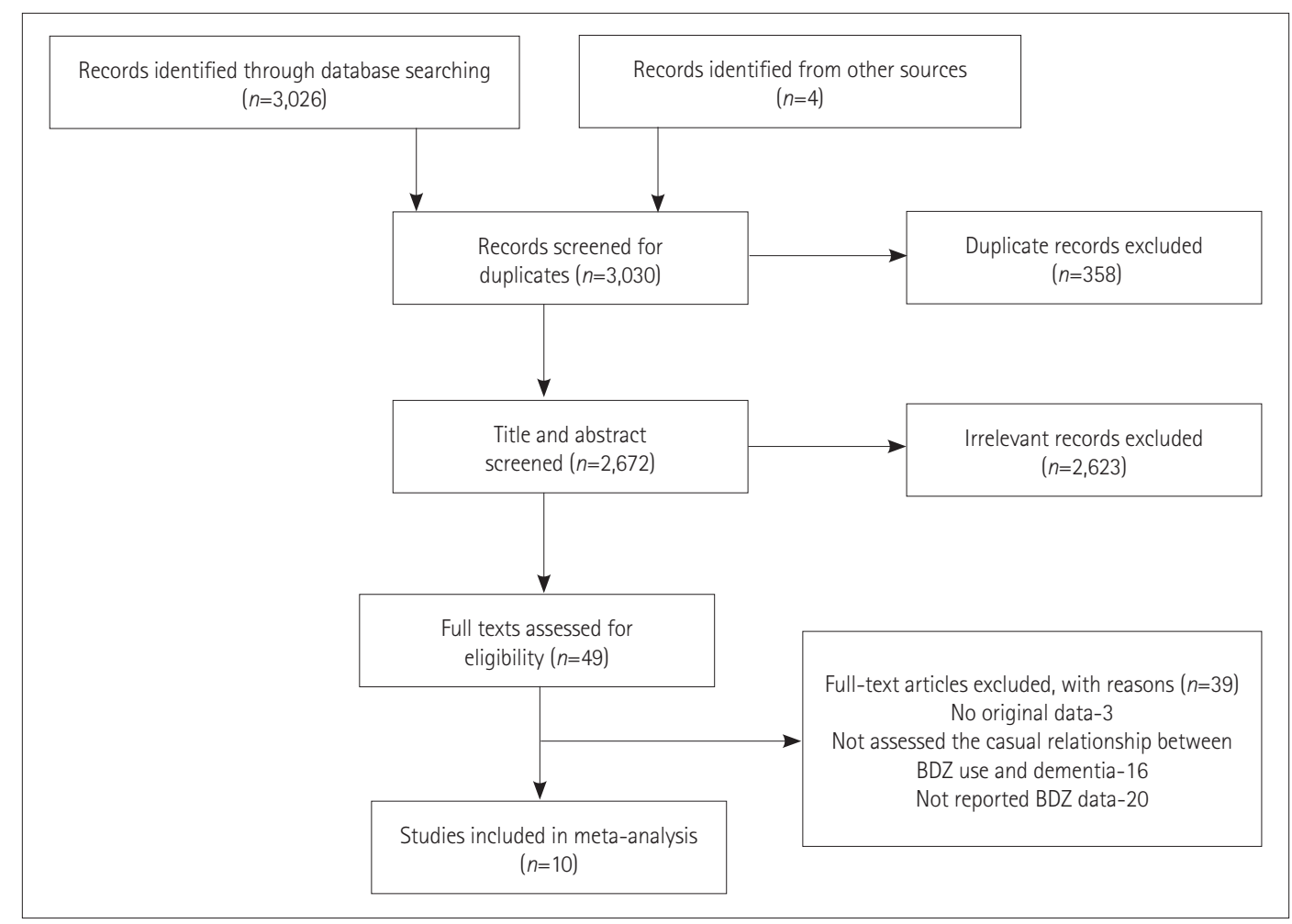

Fig. 1. PRISMA flow diagram of the study selection process. Flow chart shows the number of citations retrieved by database search and criteria used to include or exclude a citation. Number of citations excluded are given in rectangular box along with the reason for exclusion. A total of 3,030 citations were screened and out of which 10 citations were finally included for pooled analysis. BDZ: benzodiazepine, n: number of studies. 


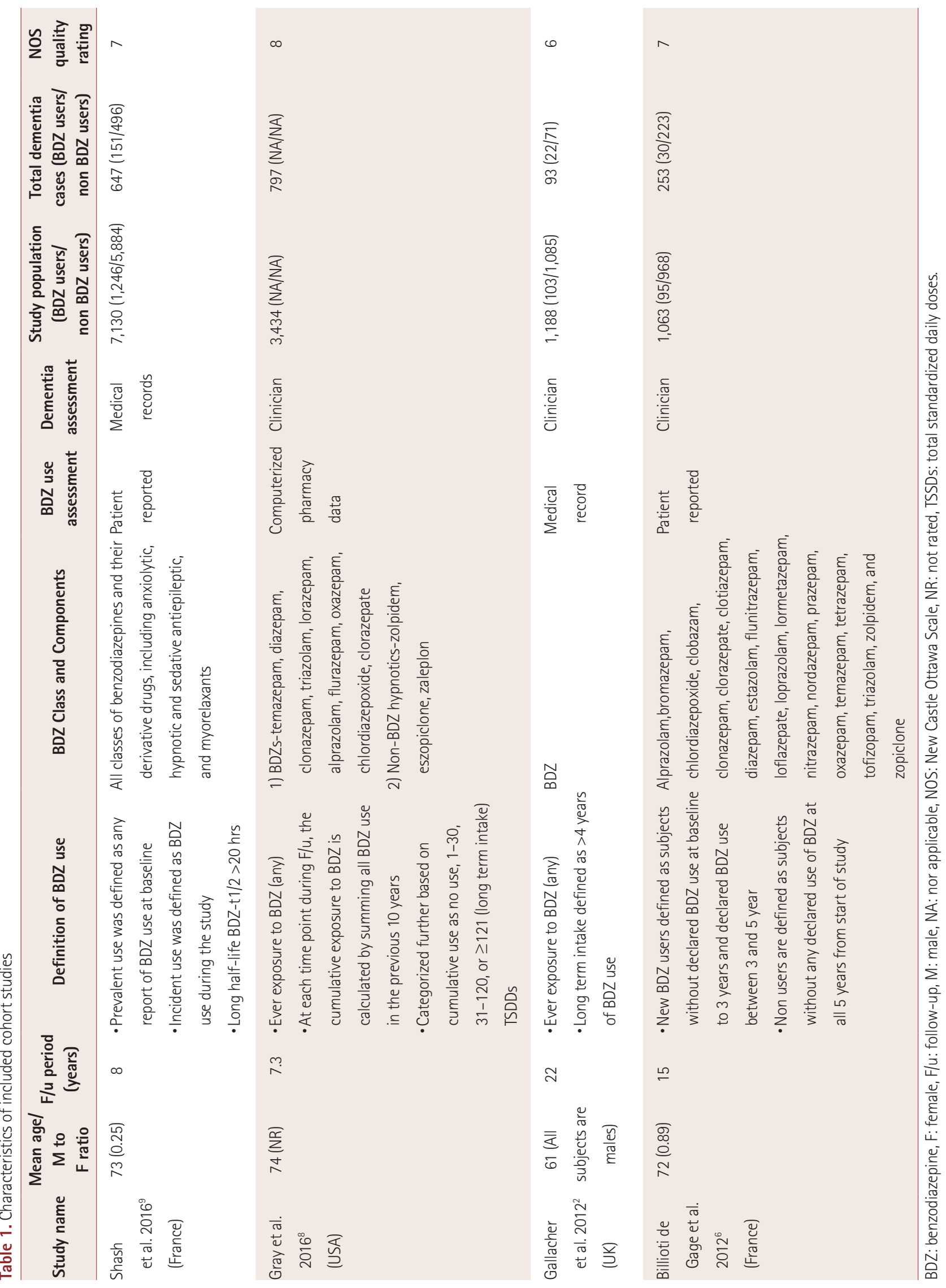




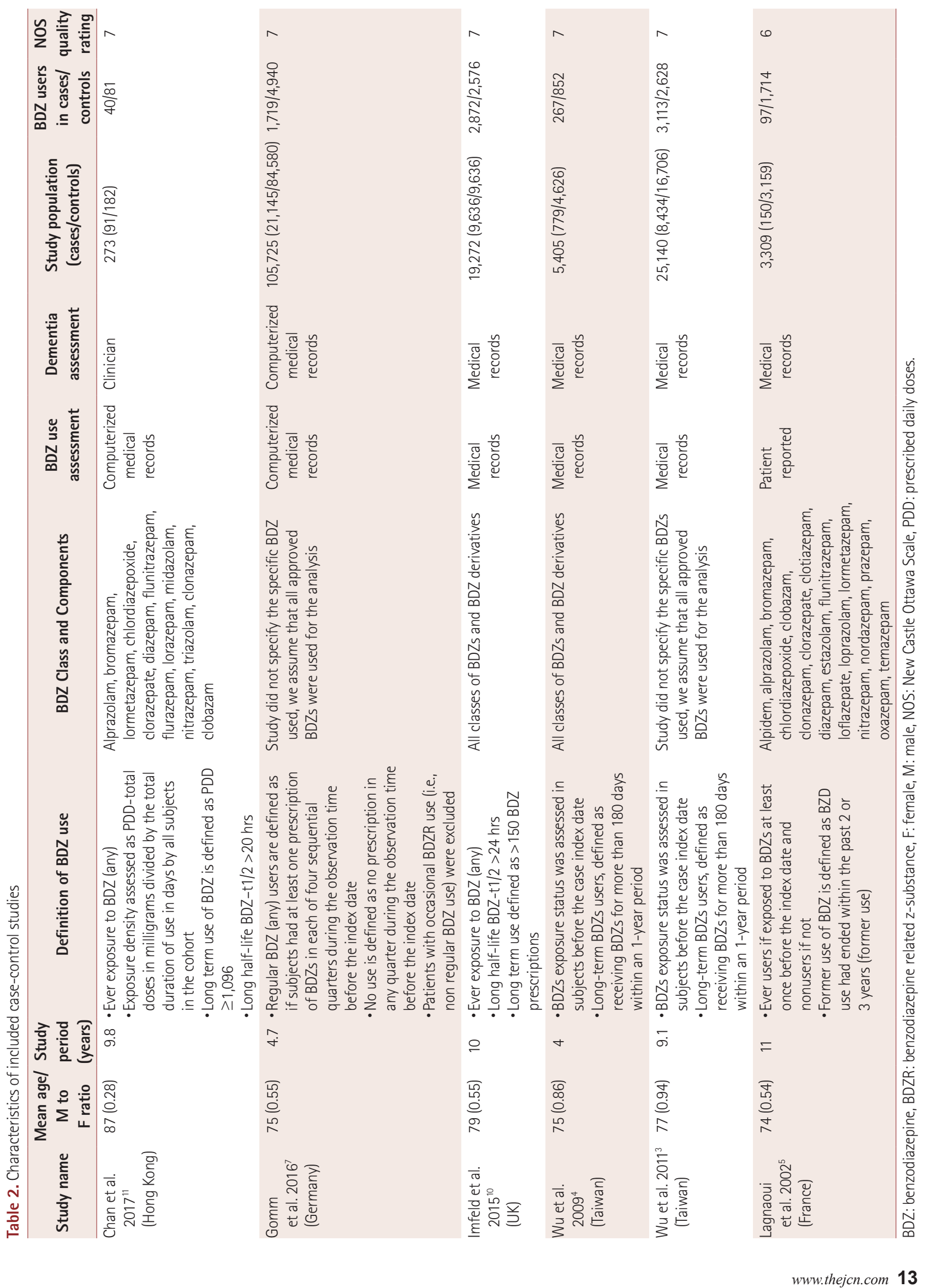


who were followed up for 4-11 years, comprising 8,108 BDZ users in 40,235 dementia cases and 12,791 BDZ users in 109,889 controls. Only one of the six case-control studies found a negative relationship between $\mathrm{BDZ}$ use and the risk of dementia. ${ }^{11}$ Two studies each were conducted in Taiwan and Europe, and one each in UK and Hong Kong (Table 2).

The 4 cohort studies were published between 2011 and 2016 and involved 12,815 participants. These studies had follow-up periods ranging from 7 to 22 years, and involved 1,790 dementia cases and more than $1,500 \mathrm{BDZ}$ users. A negative relationship between $\mathrm{BDZ}$ use and the risk of dementia was found in one study. ${ }^{9}$ The four cohort studies comprised two conducted in France and one each in the UK and USA (Table 1).

\section{Exposure to BDZ assessment in included studies}

None of the included studies focused on a single class or category of BDZ. Patients were classified as being exposed to $\mathrm{BDZ}$ if they took any BDZ for a prespecified duration. However, the included studies performed ad-hoc analyses according to various categories of BDZ. The method of exposure assessment differed across the included studies. The use of $\mathrm{BDZ}$ was analyzed using medical records in five of the six casecontrol studies, and self-reported in the sixth study, while medical records were used in two of the four cohort studies, with self-reporting in the other two. Further information about the $\mathrm{BDZ}$ use and assessment is given in Table 1 and 2.

\section{Quality assessment results}

The NOS scores resulted in eight of the ten studies being categorized as of moderate quality, and the remaining two stud- ies categorized as of low quality. 2,5 These results were as expected since all of the included studies were observational in nature and prone to selection bias. Detailed results of the quality assessment are given in the Supplementary Materials 2 (in the online-only Data Supplement). Despite the mediocre overall quality of the included studies, the present pooled meta-analysis results are reliable.

\section{Pooled RR estimates: ever BDZ use vs. never BDZ use} The heterogeneity parameter ( $p_{\text {heterogeneity }}<0.05, \mathrm{I}^{2}=97 \%$ ) was observed to be significant, and so a random-effects model was chosen over a fixed-effects model. The combined analysis of the ten studies inferred that patients with ever $\mathrm{BDZ}$ use were associated with a considerable increase in the risk of dementia $(\mathrm{RR}=1.51,95 \% \mathrm{CI}=1.17-1.95, p=0.002)$ compared to $\mathrm{pa}-$ tients with never $\mathrm{BDZ}$ use. The multivariable-adjusted RR estimate and $95 \%$ CI of each study and the pooled RR are shown in Fig. 2.

\section{Subgroup analysis}

No significant difference in the two pooled RR values was found when the studies were grouped into those of moderate quality $\left(\mathrm{RR}=1.44\right.$, Cochrane $\mathrm{Q}=302.5$, $p_{\text {heterogeneity }}<0.05, \mathrm{I}^{2}=$ $97.7 \%)$ and low quality $\left(\mathrm{RR}=1.87\right.$, Cochrane $\mathrm{Q}=1.2, p_{\text {heterogeneity }}=$ $\left.0.280, I^{2}=14.5 \%\right)\left(p_{\text {interaction }}=0.64\right)($ Fig. 3$)$, nor when the studies were grouped according to the study design (case-control and cohort studies) $\left(p_{\text {interaction }}=0.43\right)$. However, the positive relationship between $\mathrm{BDZ}$ use and the risk of dementia was stronger in the case-control studies $\left(\mathrm{RR}=1.57\right.$, Cochrane $\mathrm{Q}=294$, $p_{\text {het }}$. erogeneity $\left.<0.05, \mathrm{I}^{2}=98.3 \%\right)$ than in the cohort studies $(\mathrm{RR}=1.26$, Cochrane Q=6.4, pheterogeneity $=0.091, \mathrm{I}^{2}=53.5 \%$ ) (Fig. 4 ).

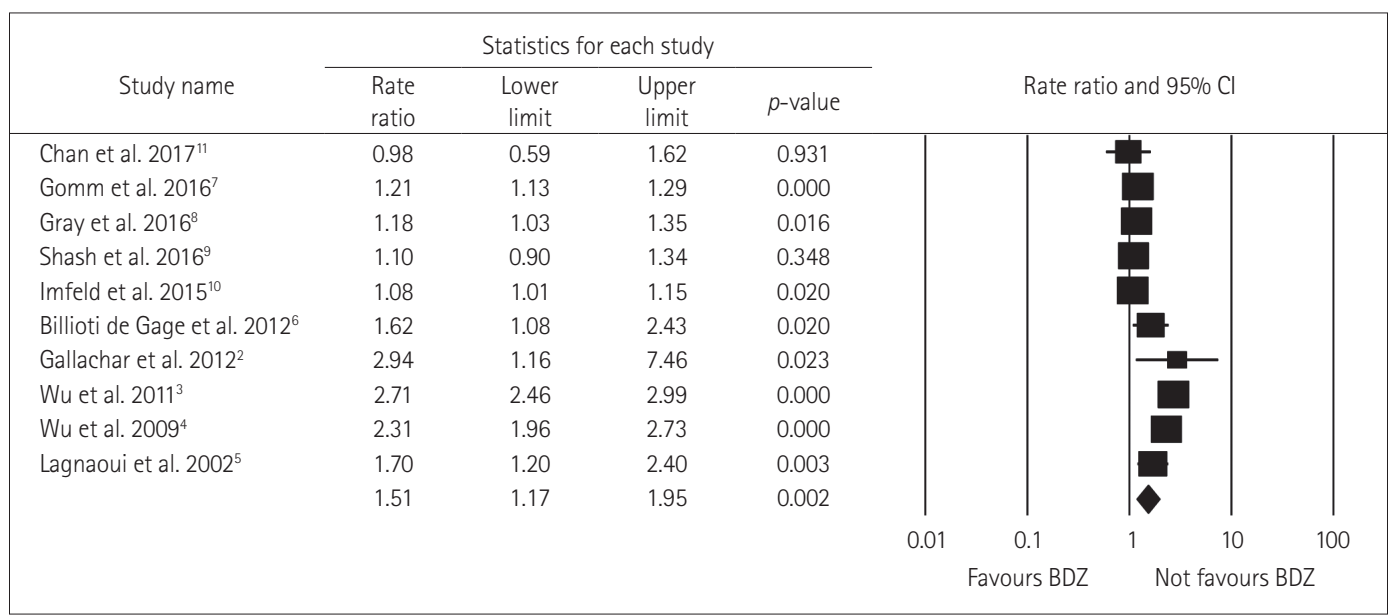

Fig. 2. Forest plot of RR of ever BDZ users compared to never BDZ users. There is an increased risk of dementia in ever BDZ users compared to never BDZ users. Forest plot representing RR estimates with 95\% Cls of each study and combined RR based on 10 studies (6 case-control and 4 cohort studies). Squares indicate RR in each study. The square size is proportional to the weight of the corresponding study in the meta-analysis; the length of horizontal lines represents the $95 \% \mathrm{Cl}$. The diamond indicates the pooled RR and 95\% Cl (random-effects model). BDZ: benzodiazepines, $\mathrm{Cl}$ : confidence interval, RR: relative risk. 


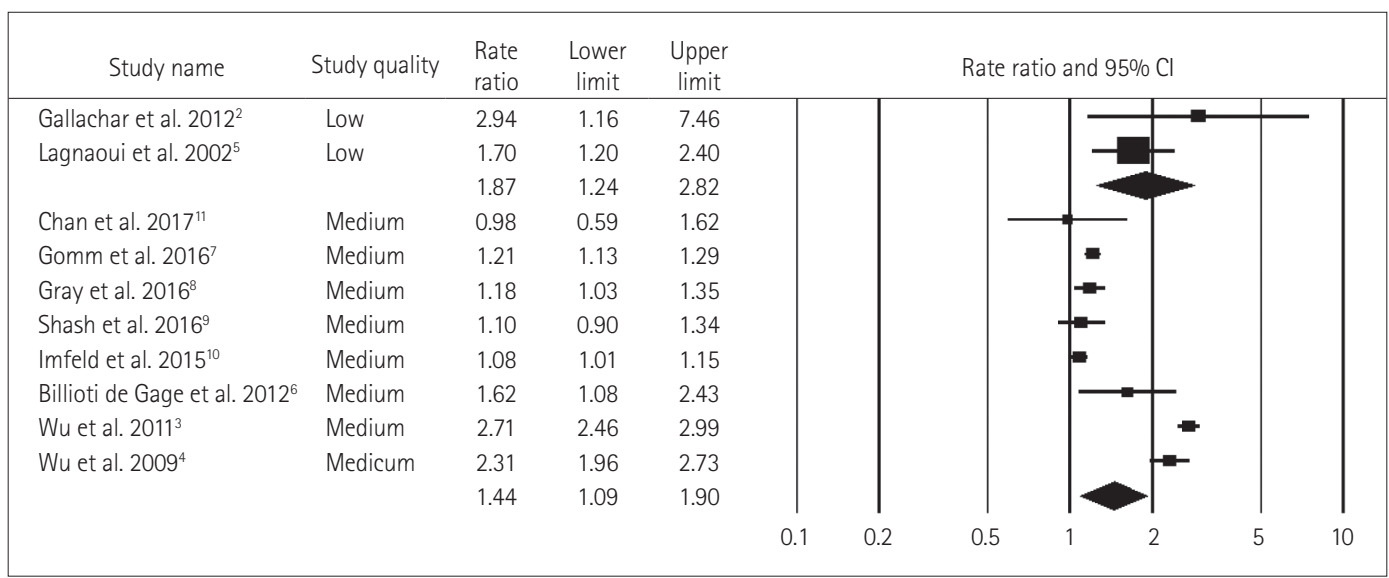

Fig. 3. Forest plot of subgroup-analysis for ever BDZ users vs. never BDZ users accrding to study quality (low and medium study quality). No significant difference in RR found among the subgroups according to medium $(n=8)$ and low $(n=2)$ quality studies. Both indicating an increased risk of dementia in ever BDZ users compared to never BDZ users. Results of each subgroup is shown at the end of each subgroup in diamond shape. Squares indicate RR in each study. The square size is proportional to the weight of the corresponding study in the meta-analysis; the length of horizontal lines represents the $95 \% \mathrm{Cl}$. BDZ: benzodiazepines, $\mathrm{Cl}$ : confidence interval, n: number of studies, RR: relative risk.

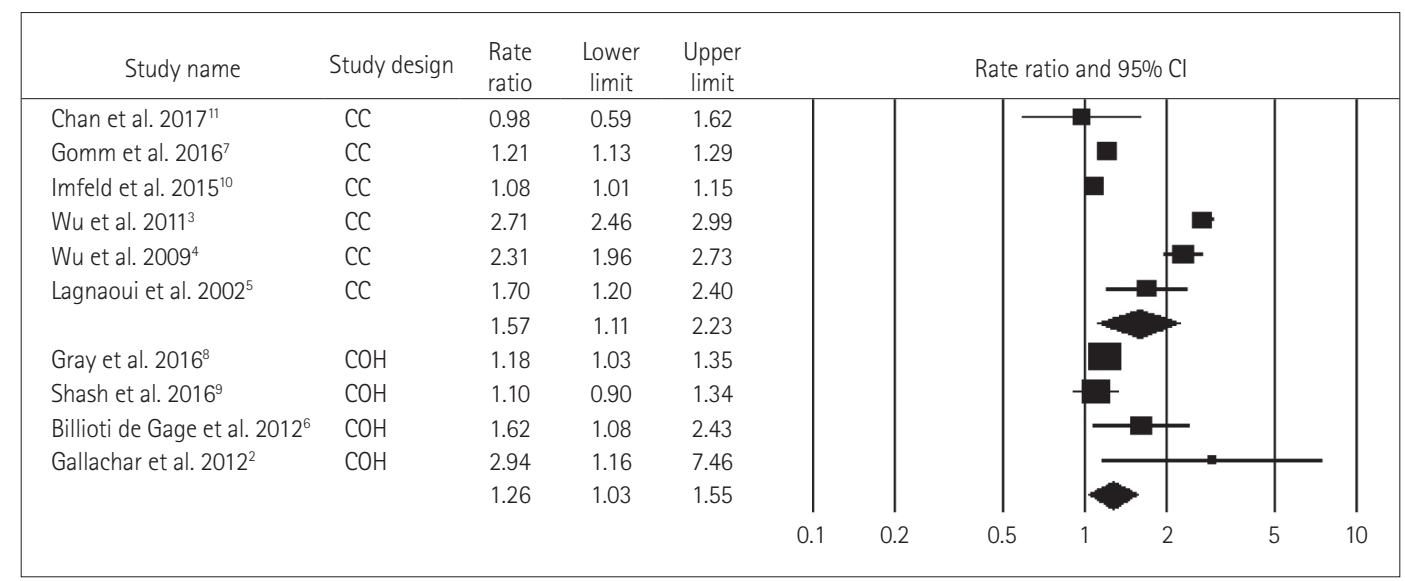

Fig. 4. Forest plot of subgroup-analysis for ever BDZ users vs. never BDZ users accrding to study design (case-control and cohort). No significant difference in RR found among the subgroups according to cohort $(n=4)$ and case-control $(n=6)$ studies. Both indicating an increased risk of dementia in ever BDZ users compared to never BDZ users. Forest plot representing pooled (random-effects model) results (RR and 95\% Cls) of subgroup-analysis according to study design i.e. Results of each subgroup is shown at the end of each subgroup in diamond shape and could be inferred as increased risk of dementia in BDZ users across the study design. Squares indicate RR in each study. The square size is proportional to the weight of the corresponding study in the meta-analysis; the length of horizontal lines represents the 95\% Cl. BDZ: benzodiazepines, CC: case-control, $\mathrm{Cl}$ : confidence interval, $\mathrm{COH}$ : Cohort, n: number of studies, RR: relative risk.

\section{Long-term BDZ use}

The long-term use of $\mathrm{BDZ}$ and the risk of dementia were assessed in four studies.,4,8,10 Long-term BDZ use was significantly associated with an increased risk of dementia $(\mathrm{RR}=$ 1.21 , Cochrane $\mathrm{Q}=3.4, p_{\text {heterogeneity }}=0.330, \mathrm{I}^{2}=12.7 \%$ ) when compared to the short-term use of BDZ (Fig. 5).

\section{Use of long-acting BDZ}

The use of long-acting $\mathrm{BDZ}$ and the risk of dementia were assessed in four studies. ${ }^{79-11}$ The use of long-acting BDZ was not strongly related to the risk of dementia $(\mathrm{RR}=1.16$, Cochrane $\left.\mathrm{Q}=13.3, p_{\text {heterogeneity }}<0.05, \mathrm{I}^{2}=77.6 \%\right)$ when compared to patients taking short- or medium-term-acting BDZ (Fig. 6).

\section{Publication bias}

The $p$ values for Begg's $(p=0.21$ ) and Egger's $(p=0.49)$ tests indicated that publication bias was not present, and the visual inspection of the funnel plot also did not reveal any asymmetry (Fig. 7).

\section{Sensitivity analysis}

The robustness of the analysis results was tested by performing a sensitivity analysis in which the overall effect size was measured by removing one study at a time. This analysis produced no significant variation in the pooled RR when excluding either the outlier study of Chan et al. ${ }^{11}$ (due to a very small sample) ( $\mathrm{RR}=1.57,95 \% \mathrm{CI}=1.20-2.06)$ or any of the 
Risk of Dementia in Long Term Benzodiazepines Users

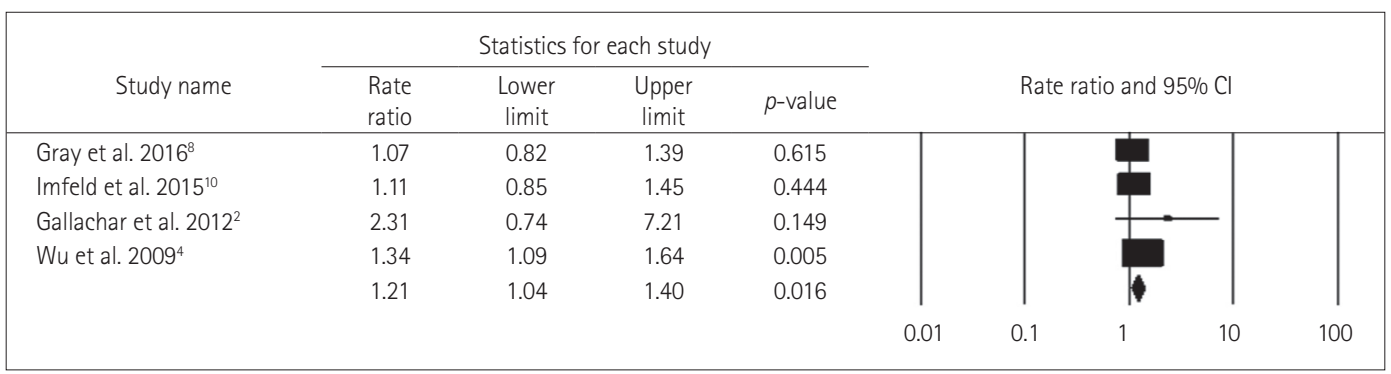

Fig. 5. Forest plot of secondary-analysis for long term BDZ users compared to short term BDZ users. Long term BDZ users have significantly higher risk of dementia compared to short term BDZ users. Forest plot representing pooled estimate of RR and 95\% Cls based duration of BDZ use. Squares indicate RR in each study. The square size is proportional to the weight of the corresponding study in the meta-analysis; the length of horizontal lines represents the $95 \% \mathrm{Cl}$. The diamond indicates the pooled RR and 95\% Cl (random-effects model). BDZ: benzodiazepines, $\mathrm{Cl}$ : confidence interval, RR: relative risk.

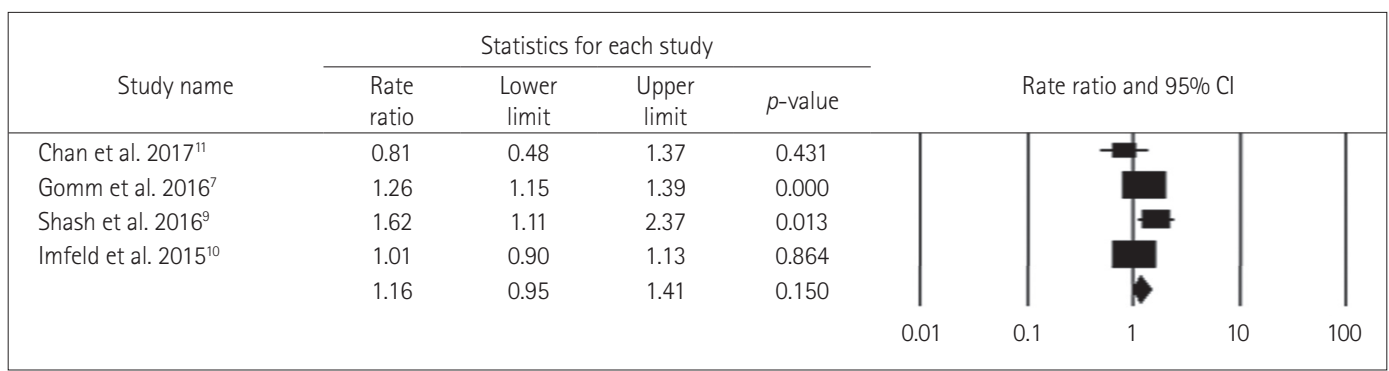

Fig. 6. Forest plot of secondary-analysis for long acting BDZ users compared to short/medium acting BDZ users. Long acting BDZ users have non significantly higher risk of dementia compared to short acting BDZ users. Forest plot representing pooled estimate of RR and 95\% Cls based halflife of BDZ. Squares indicate RR in each study. The square size is proportional to the weight of the corresponding study in the meta-analysis; the length of horizontal lines represents the 95\% Cl. The diamond indicates the pooled RR and 95\% Cl (random-effects model). BDZ: benzodiazepines, $\mathrm{Cl}$ : confidence interval, RR: relative risk.

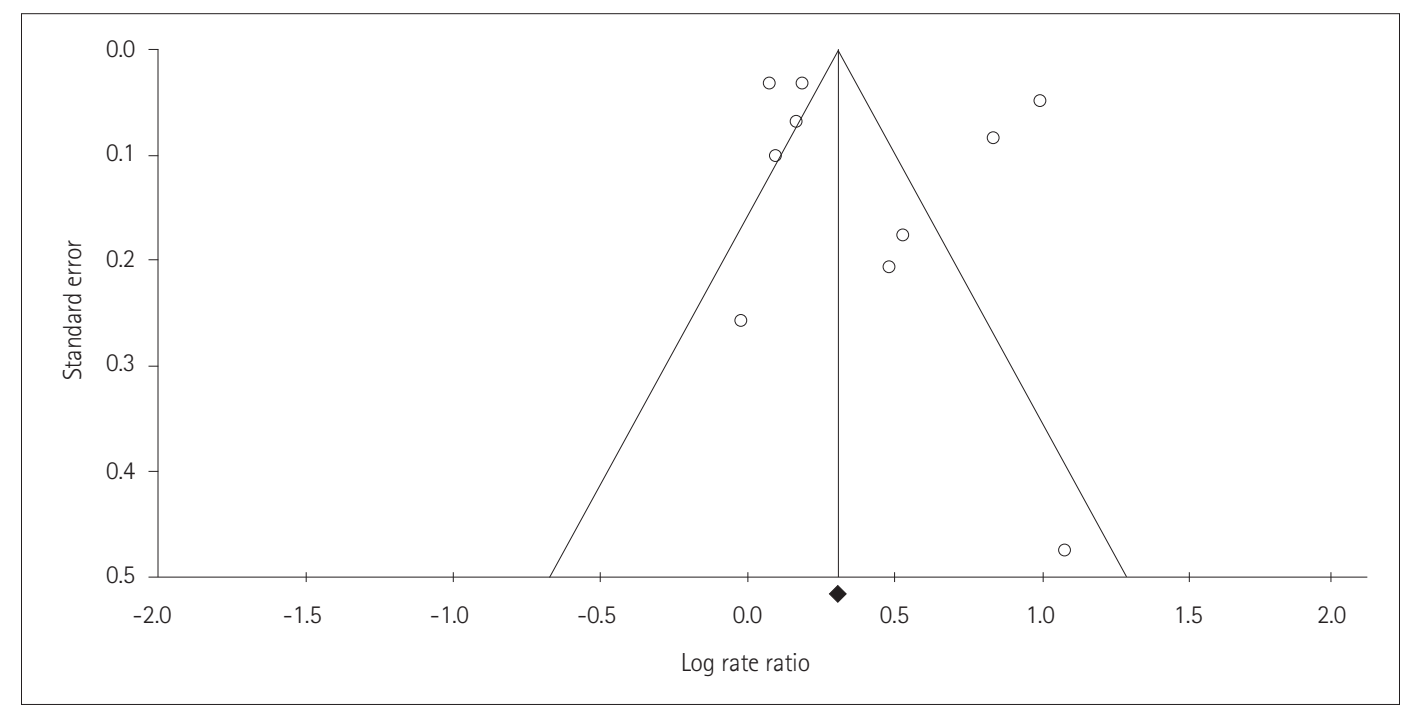

Fig. 7. Funnel plot representing publication bias. Funnel plot does not have any asymmetry representing no significant publication bias in the study. Rate ratios are displayed on a logarithmic scale. Circles represent studies included in the meta-analysis.

other studies $(\mathrm{RR}=1.37-1.58)$, thereby confirming the robustness of the present results (Fig. 8).

\section{DISCUSSION}

This meta-analysis pooled ten studies and found that BDZ significantly increases the risk of dementia in the elderly population. This effect was greater in patients using BDZ with a longer half-life ( $>20$ hours half-life) and taking BDZ for a longer duration ( $>3$ years).

The positive relationship between $\mathrm{BDZ}$ use and the risk of dementia was stronger in the case-control studies $(R R=1.57)$

16 J Clin Neurol 2019;15(1):9-19 


\begin{tabular}{|c|c|c|c|c|c|c|c|c|c|c|}
\hline \multirow{3}{*}{$\begin{array}{l}\text { Study name } \\
\text { Imfeld et al. } 2015^{10}\end{array}$} & \multicolumn{4}{|c|}{ Statistics for study removed } & \multirow{2}{*}{\multicolumn{6}{|c|}{ Rate ratio $(95 \% \mathrm{Cl})$ with study removed }} \\
\hline & \multirow{2}{*}{$\begin{array}{r}\text { Point } \\
1.58\end{array}$} & \multirow{2}{*}{$\begin{array}{c}\begin{array}{c}\text { Lower } \\
\text { limit }\end{array} \\
1.17\end{array}$} & \multirow{2}{*}{$\begin{array}{c}\begin{array}{c}\text { Upper } \\
\text { limit }\end{array} \\
2.14\end{array}$} & $p$-value & & & & & & \\
\hline & & & & 0.003 & & & & & & \\
\hline Shash et al. $2016^{9}$ & 1.57 & 1.19 & 2.08 & 0.001 & & & & & & \\
\hline Chan et al. $2017^{11}$ & 1.57 & 1.20 & 2.06 & 0.001 & & & & & & \\
\hline Gomm et al. $2016^{7}$ & 1.56 & 1.12 & 2.18 & 0.008 & & & & & & \\
\hline Gray et al. $2016^{8}$ & 1.56 & 1.17 & 2.08 & 0.002 & & & & & & \\
\hline Billioti de Gage et al. $2012^{6}$ & 1.50 & 1.14 & 1.97 & 0.003 & & & & & & \\
\hline Lagnaoui et al. $2002^{5}$ & 1.49 & 1.14 & 1.96 & 0.004 & & & & & & \\
\hline Gallachar et al. $2012^{2}$ & 1.46 & 1.13 & 1.90 & 0.005 & & & & & & \\
\hline Wu et al. $2009^{4}$ & 1.43 & 1.10 & 1.87 & 0.008 & & & & & & \\
\hline \multirow[t]{4}{*}{ Wu et al. $2011^{3}$} & 1.37 & 1.16 & 1.62 & 0.000 & & & & & & \\
\hline & 1.51 & 1.17 & 1.95 & 0.002 & & & & & & \\
\hline & & & & & \multirow[t]{2}{*}{0.1} & 0.2 & 0.5 & 1 & 2 & 10 \\
\hline & & & & & & Favo & SBDZ & & & ars BDZ \\
\hline
\end{tabular}

Fig. 8. Sensitivity analysis forest plot. There is no significant impact of single study on pooled effect estimate. Sensitivity analysis plot representing impact on pooled effect estimate if individual studies are removed one by one based on ascending order of difference between the effect size and the pooled estimate. Square and length of horizontal lines beside each study represents pooled effect estimate and its $95 \% \mathrm{Cl}$ of all included studies except the study beside the square. The diamond indicates the pooled RR and 95\% Cl (random-effects model). BDZ: benzodiazepines, $\mathrm{Cl}$ : confidence interval, RR: relative risk.

than in the cohort studies $(\mathrm{RR}=1.26)$. Such a trend is usually observed when effect estimates for adverse effects are compared between case-control and cohort studies. A methodological review performed by Golder et al. ${ }^{14}$ found that the estimates for adverse effects were slightly larger (although not significantly) for case-control than cohort studies. It is generally considered that case-control studies have a higher risk of bias than cohort studies and are more susceptible to selection and recall bias.

The RR values for long-term $\mathrm{BDZ}$ use (=1.21) and the use long-acting $\mathrm{BDZ}(=1.16)$ were lower than the primary pooled $\mathrm{RR}$ (ever $\mathrm{BDZ}$ user vs. never $\mathrm{BDZ}$ user; $\mathrm{RR}=1.51$ ) estimate because the comparator to estimate RR differed for the three estimates, which meant they could not be compared.

The long-term use of $\mathrm{BDZ}$ is associated with the accumulation of generalized cognitive deficits that lead to an increased risk of dementia in long-term BDZ users compared to shortterm $\mathrm{BDZ}$ users. Withdrawal symptoms could be observed in short-term BDZ users, but no accumulation of cognitive deficits. The trend observed in the present analysis is consistent with the explanation given above.

This studies included the following three categories of BDZ half-life values: 1 ) short-acting BDZs (half-life $<12 \mathrm{~h}$ ) such as midazolam and triazolam, 2) intermediate-acting BDZs (halflife $=12-24 \mathrm{~h}$ ) such as alprazolam, clonazepam, lorazepam, oxazepam, temazepam, lormetazepam, and flunitrazepam, and 3) long-acting BDZs (half-life $>24 \mathrm{~h}$ ) such as chlordiazepoxide, flurazepam, diazepam, nitrazepam, and quazepam. The pooled analysis showed that the use of long-acting BDZ is associated with a nonsignificant increase in the risk of de- mentia compared to patients taking short- or medium-termacting BDZ.

The results of the meta-analysis should be interpreted carefully since statistically significant heterogeneity was observed in the included studies. The source of heterogeneity in the pooled studies was not revealed in the predefined subgroup analysis. However, the study characteristics in Table 1 and 2 provide interesting insights that might explain the observed heterogeneity. The sample sizes of the included studies ranged widely, from 273 to 105,725 , and studies with smaller samples had effect estimates with wider 95\% CIs or RRs of less than 1. Pooling studies with differing sample sizes might lead to heterogeneity. However, other sources of heterogeneity should not be ignored, including the definition of $\mathrm{BDZ}$ exposure and the diagnostic method used to confirm the presence of dementia in the included studies.

A few recently published studies have found no association between BDZ and dementia. Despite this, the pooled evidence shows that risk of dementia is high in patients taking BDZ. ${ }^{10,11}$ Three associated processes might have impacted the development of dementia in patients using BDZ. One characteristic is that $\mathrm{BDZ}$ reduces the level of beta-site amyloid precursor protein-cleaving enzyme 1 and the c-secretase activity that subsequently slows down the buildup of amyloid-beta oligomers in the brain. ${ }^{15,16}$ Such a possible positive effect along with an antiglutamatergic action of BDZ has never been confirmed. ${ }^{17}$ Another possible influencing process is the presence of astrocytes at the amyloid plaques in patients with predementia lesions having GABA-secreting activity, which will enhance the deleterious cognitive effects of $\mathrm{BDZ} .^{18}$ 
Another major process via which BDZs might result in dementia is decreasing the brain activation level. ${ }^{19}$

The association between BDZs and dementia could be a reverse-causation bias, since the main indications for $\mathrm{BDZ}$ (insomnia and anxiety) can also be prodromal of dementia disorders. ${ }^{6}$ The main hurdle with observational studies is collecting data to support the possibility of reverse causation over a long period. ${ }^{20}$

However, we suggest that bias due to reverse causation was highly unlikely in the present analysis, since BDZs were taken for more than 10 years before the diagnosis of dementia. ${ }^{2}$ The relationship between $\mathrm{BDZ}$ use and dementia can be considered a marker of the increased risk of the occurrence of dementia, and not as the main cause. However, some studies have recommended that before diagnosing dementia, data from previous years should be analyzed to see if there is a strong correlation between the symptoms of dementia and $\mathrm{BDZ}$ prescriptions. ${ }^{21-23}$ Such an association can be inferred as a confounding factor based on indication and reverse causation. If reverse causation is a factor, the association of $\mathrm{BDZ}$ with dementia should be stronger in short-term users than in long-term past users. Imfeld et al. ${ }^{10}$ suggested that in patients using BDZs for $<1$ year before a diagnosis have an increased risk of Alzheimer's and vascular dementia compared with patients using BDZs for 2-4 years before a dementia diagnosis.

The Caerphilly Prospective Study of Gallacher et al. ${ }^{2}$ found a significant positive association between $\mathrm{BDZ}$ use and dementia. Those authors also reported that the relationship was strongest for cumulative use over $>4$ years $(\mathrm{OR}=4.38,95 \%$ $\mathrm{CI}=1.15-16.75)$. The very long follow-up was an undisputable strength of the present study, even if its low statistical power precluded several subanalyses for assessing the plausibility of reverse causation.

In addition to an increased risk of dementia, there is also evidence that $\mathrm{BDZs}$ increase the risk of injurious falls, fractures, acute respiratory failure, and delirium, all of which reduce a patient's quality of life and increases the financial burden. ${ }^{24-26}$ The ten studies published on the topic were not consistent, with one inferring a protective effect of $\mathrm{BDZ},{ }^{27}$ nine finding an increase in dementia disorders in $\mathrm{BDZ}$ users, ${ }^{2-6,28,29}$ and the tenth finding no relationship. ${ }^{10}$ These findings suggest that $\mathrm{BDZs}$ should be prescribed only when there is an utmost need for treatment.

A BDZ still might be the drug of choice for treating insomnia in elderly patients, with careful selection of the particular BDZ according to clinical guidelines. Short-acting BDZs should be prescribed as a short-term therapy and progressively withdrawn to avoid cumulative effects such as deceased cognition.

This study was subject to some limitations: 1 ) all of includ- ed studies were observational, which may lead to recall bias, 2) few data on the duration of action of BDZs were included in the studies, and 3) the specific roles of individual BDZs and doses could not be determined since the required data were not reported.

In conclusion, this meta-analysis has yielded evidence that $\mathrm{BDZ}$ use is associated with dementia. This association is stronger in people using long-acting BDZs for longer durations. We suggest that ultra-short-acting BDZs should be prescribed and then tapered off while using other therapies in order to avoid dependence and other long-term adverse events. Further prospective long-term studies are required to eliminate reverse-causation bias and to confirm the presence of an association between $\mathrm{BDZ}$ and dementia.

\section{Supplementary Materials}

The online-only Data Supplement is available with this article at https://doi.org/10.3988/jcn.2019.15.1.9.

\section{Conflicts of Interest}

The authors have no financial conflicts of interest.

\section{REFERENCES}

1. Griffin CE 3rd, Kaye AM, Bueno FR, Kaye AD. Benzodiazepine pharmacology and central nervous system-mediated effects. Ochsner J 2013;13:214-223.

2. Gallacher J, Elwood P, Pickering J, Bayer A, Fish M, Ben-Shlomo Y. Benzodiazepine use and risk of dementia: evidence from the caerphilly prospective study (CaPS). J Epidemiol Community Health 2012;66: 869-873.

3. Wu CS, Ting TT, Wang SC, Chang IS, Lin KM. Effect of benzodiazepine discontinuation on dementia risk. Am J Geriatr Psychiatry 2011; 19:151-159.

4. Wu CS, Wang SC, Chang IS, Lin KM. The association between dementia and long-term use of benzodiazepine in the elderly: nested case-control study using claims data. Am J Geriatr Psychiatry 2009;17: 614-620.

5. Lagnaoui R, Bégaud B, Moore N, Chaslerie A, Fourrier A, Letenneur $\mathrm{L}$, et al. Benzodiazepine use and risk of dementia: a nested case-control study. J Clin Epidemiol 2002;55:314-318.

6. Billioti de Gage S, Bégaud B, Bazin F, Verdoux H, Dartigues JF, Pérès $\mathrm{K}$, et al. Benzodiazepine use and risk of dementia: prospective population based study. BMJ 2012;345:e6231.

7. Gomm W, von Holt K, Thomé F, Broich K, Maier W, Weckbecker K, et al. Regular benzodiazepine and $\mathrm{z}$-substance use and risk of dementia: an analysis of German claims data. J Alzheimers Dis 2016;54:801808.

8. Gray SL, Dublin S, Yu O, Walker R, Anderson M, Hubbard RA, et al. Benzodiazepine use and risk of incident dementia or cognitive decline: prospective population based study. BMJ 2016;352:i90.

9. Shash D, Kurth T, Bertrand M, Dufouil C, Barberger-Gateau P, Berr C, et al. Benzodiazepine, psychotropic medication, and dementia: a population-based cohort study. Alzheimers Dement 2016;12:604-613.

10. Imfeld P, Bodmer M, Jick SS, Meier CR. Benzodiazepine use and risk of developing Alzheimer's disease or vascular dementia: a case-control analysis. Drug Saf 2015;38:909-919.

11. Chan TT, Leung WC, Li V, Wong KW, Chu WM, Leung KC, et al. Association between high cumulative dose of benzodiazepine in Chi- 
nese patients and risk of dementia: a preliminary retrospective casecontrol study. Psychogeriatrics 2017;17:310-316.

12. Altman DG, Bland JM. Interaction revisited: the difference between two estimates. BMJ 2003;326:219.

13. Begg CB, Mazumdar M. Operating characteristics of a rank correlation test for publication bias. Biometrics 1994;50:1088-1101.

14. Golder S, Loke YK, Bland M. Comparison of pooled risk estimates for adverse effects from different observational study designs: methodological overview. PLoS One 2013;8:e71813.

15. Imbimbo BP, Giardina GA. $\gamma$-secretase inhibitors and modulators for the treatment of Alzheimer's disease: disappointments and hopes. Curr Top Med Chem 2011;11:1555-1570.

16. Doody RS, Raman R, Farlow M, Iwatsubo T, Vellas B, Joffe S, et al. A phase 3 trial of semagacestat for treatment of Alzheimer's disease. $N$ Engl J Med 2013;369:341-350.

17. May PC, Robison PM. GYKI 52466 protects against non-NMDA receptor-mediated excitotoxicity in primary rat hippocampal cultures. Neurosci Lett 1993;152:169-172.

18. Jo S, Yarishkin O, Hwang YJ, Chun YE, Park M, Woo DH, et al. GABA from reactive astrocytes impairs memory in mouse models of Alzheimer's disease. Nat Med 2014;20:886-896.

19. Pariente A, de Gage SB, Moore N, Bégaud B. The benzodiazepine-dementia disorders link: current state of knowledge. CNS Drugs 2016; 30:1-7.

20. Zhang Y, Zhou XH, Meranus DH, Wang L, Kukull WA. Benzodiazepine use and cognitive decline in elderly with normal cognition. Alzheimer Dis Assoc Disord 2016;30:113-117.

21. Amieva H, Le Goff M, Millet X, Orgogozo JM, Pérès K, BarbergerGateau P, et al. Prodromal Alzheimer's disease: successive emergence of the clinical symptoms. Ann Neurol 2008;64:492-498.

22. Taragano FE, Allegri RF, Krupitzki H, Sarasola DR, Serrano CM, Loñ L, et al. Mild behavioral impairment and risk of dementia: a prospective cohort study of 358 patients. J Clin Psychiatry 2009;70:584-592.

23. Lyketsos CG, Lopez O, Jones B, Fitzpatrick AL, Breitner J, DeKosky S. Prevalence of neuropsychiatric symptoms in dementia and mild cognitive impairment: results from the cardiovascular health study. JAMA 2002;288:1475-1483.

24. Pariente A, Dartigues JF, Benichou J, Letenneur L, Moore N, Fourrier-Réglat A. Benzodiazepines and injurious falls in community dwelling elders. Drugs Aging 2008;25:61-70.

25. Rossat A, Fantino B, Bongue B, Colvez A, Nitenberg C, Annweiler C, et al. Association between benzodiazepines and recurrent falls: a crosssectional elderly population-based study. J Nutr Health Aging 2011; 15:72-77.

26. Díaz-Gutiérrez MJ, Martínez-Cengotitabengoa M, Sáez de Adana E, Cano AI, Martínez-Cengotitabengoa MT, Besga A, et al. Relationship between the use of benzodiazepines and falls in older adults: a systematic review. Maturitas 2017;101:17-22.

27. Fastbom J, Forsell Y, Winblad B. Benzodiazepines may have protective effects against Alzheimer disease. Alzheimer Dis Assoc Disord 1998;12: 14-17.

28. Chen PL, Lee WJ, Sun WZ, Oyang YJ, Fuh JL. Risk of dementia in patients with insomnia and long-term use of hypnotics: a populationbased retrospective cohort study. PLoS One 2012;7:e49113.

29. Billioti de Gage S, Moride Y, Ducruet T, Kurth T, Verdoux H, Tournier M, et al. Benzodiazepine use and risk of Alzheimer's disease: casecontrol study. BMJ 2014;349:g5205. 